\title{
MINING roX1 RNA IN Drosophila GENOMES USING COVARIANCE MODELS
}

\author{
K. Byron $,{ }^{*},{ }^{* *},{ }^{* * * *}$ M.C.-Cervantes, ${ }^{*},{ }^{* * *}$ J.T.L. Wang, ${ }^{*},{ }^{* *},{ }^{* * *}$ W.C. Lin,${ }^{* * * *}$ and Y. Park ${ }^{* * * * *}$
}

\begin{abstract}
Evolutionarily conserved functional domains of non-coding RNA on chromosome X (roX1) have been identified in eight Drosophila species in a prior study. Among our findings, three GUUNUACG repeats were localized in the $3^{\prime}$-end of the predicted roX1 RNAs for these Drosophila species. In this study, we use a covariance model $(\mathrm{CM})$ to search for the characteristic features of roX1 functional domains as a way to predict new examples of these structured RNAs in other Drosophila species, as sequencing data become available. We scan whole genomes of Drosophila and identify search results in available "region" terms, i.e., "chromosome" or "scaffold", depending on the annotation status of the particular species being surveyed. With known roX1 examples produced through our prior studies for support in comparative analysis, we hypothesize that it is possible to predict novel roX1 functional domains accurately from sequence information alone. Annotating roX1 on a genomic scale provides insight into evolutionary processes and phylogenetic relationships among the analyzed species. Based on our results, we confirmed that a $\mathrm{CM}$ search is effective in mining roX1 RNA genes and, that due to its inherent flexibility, this mining approach will likely prove successful for similar endeavours in various other organisms.
\end{abstract}

\section{Key Words}

Non-coding RNA, covariance model, secondary structure, Drosophila, comparative genomics

\section{Introduction}

Non-coding RNAs (ncRNAs) are functional RNA transcripts that are not translated into protein (i.e., they are not messenger RNAs). Recent research has shown that

* Bioinformatics Program, New Jersey Institute of Technology, Newark, NJ 07102, USA; e-mail: byron@njit.edu

** Department of Computer Science, New Jersey Institute of Technology, Newark, NJ 07102, USA; e-mail: wangj@njit.edu

*** Department of Biological Sciences, Rutgers University, Newark, NJ 07102, USA; e-mail: miguelcc@andromeda. rutgers.edu

**** Institute of Biomedical Sciences, Academia Sinica, Taipei, Taiwan, Republic of China; e-mail: wenlin@ibms.sinica.edu.tw

***** Department of Cell Biology and Molecular Medicine, University of Medicine and Dentistry of New Jersey - New Jersey Medical School, Newark, NJ 07103, USA; e-mail: parky1@umdnj.edu

(paper no. 210-1012)
ncRNAs perform a wide range of functions in the cell [1-4]. RNA on X (roX1) plays an essential role in equalizing the level of transcription on the $\mathrm{X}$ chromosome in Drosophila males (XY) to that of females (XX) [5]. Experiments have confirmed that roX1 RNA exists in eight Drosophila species [6-8]. This leads to the hypothesis that there exists secondary structure conservation of the roX 1 gene among other Drosophila species $[7,8]$ for which specific roX1 genomic coordinates are as yet unknown. Recent advances in the research of genomes from 12 Drosophila species [9] might contribute to support this hypothesis.

The covariance model (CM) method Infernal, used in the prediction of functional domain conservation in ncRNAs, is considered by experts to be one of the most accurate general tools [10]. A CM is a statistical representation or profile of a family of related RNAs that share a common consensus secondary structure [11]. The Infernal software package [12-14] contains a utility, cmbuild, for creating a $\mathrm{CM}$ from an alignment of sequences in the Stockholm format, and a utility, cmsearch, to search for sequences that are similar to the model. The cmsearch process is computationally expensive when a single-processor approach is used. However, by utilizing a parallel processing approach, search results can be obtained in efficient time frames.

We began by demonstrating the capability of using a $\mathrm{CM}$ in a genome-scale homology search. We obtained roX1 sequences from eight species of Drosophila for which experimental evidence of ro $X 1$ has been found, namely: $D$. ananassae, D. erecta, D. melanogaster, D. mojavensis, D. pseudoobscura, D. simulans, D. virilis and D. yakuba [7]. Subsequently, we focused on locating evidence of roX1 sequences on the complete genomes of these eight species using a CM derived from our roX1 sample sequences. We expected this search to be successful and it was in 6 of the 8 species. This was critical to demonstrate as a "proof of concept" that our method would find that which we know exist from empirical data. Subsequently, we used the same CM to search for evidence of these conserved structures in the complete genomes of the four remaining sequenced Drosophila species for which there are no transcript-derived roX1 sequences. These four species are D. grimshawi, D. persimilis, D. sechellia and D. willistoni. Such a comparative genomics approach has been successful in the unicellular organism Saccharomyces cerevisiae [15]. 


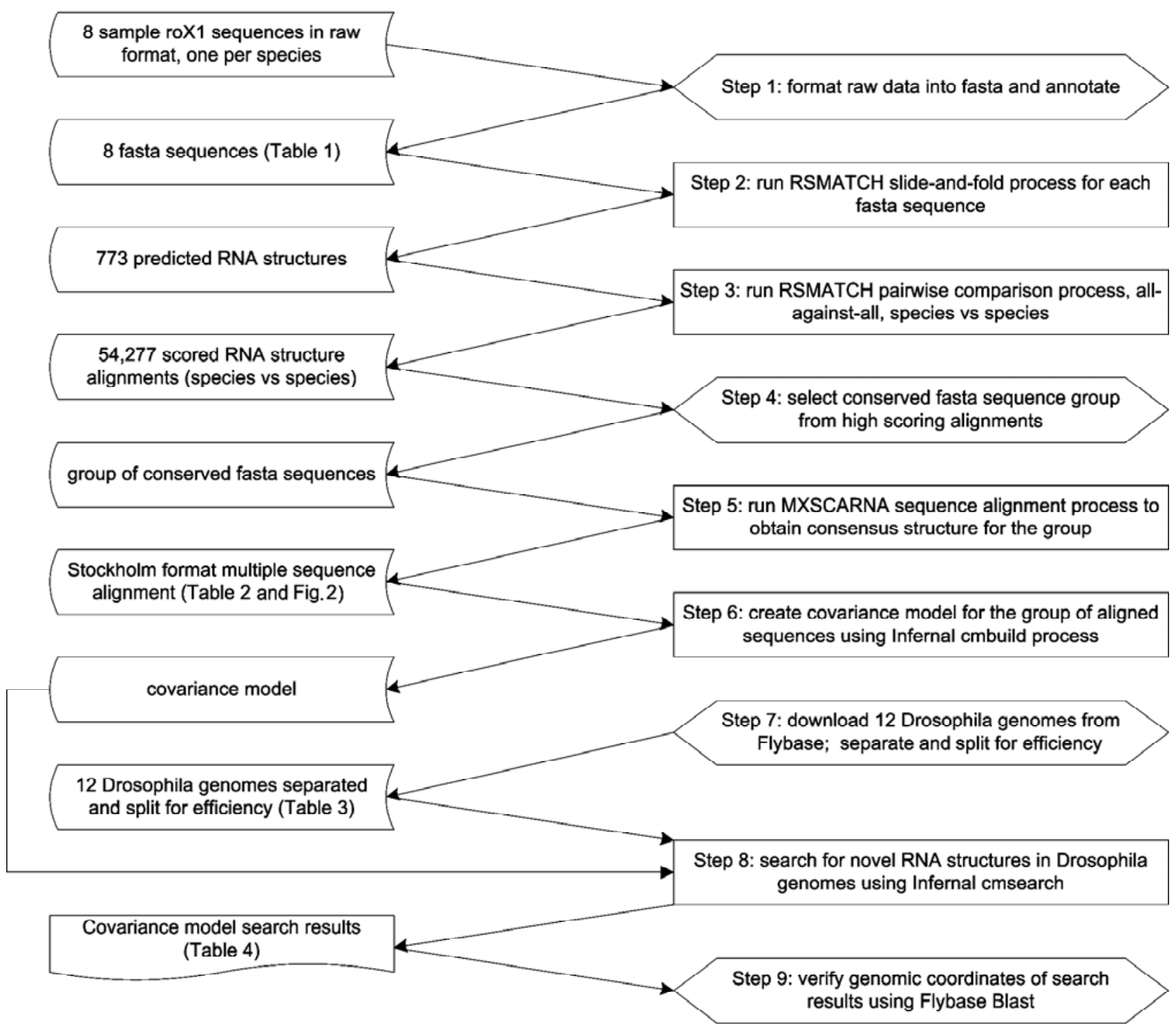

Figure 1. Drosophila roX1 data mining process utilizing covariance model methodology. See methods section for description.

Using this approach, our results show strong evidence of the presence of roX1 functional domains in the genome of $D$. sechellia. We believe this finding to be novel and significant in ongoing genomic studies of Drosophila and related taxonomic groups. This bioinformatics study lays the groundwork for future accurate and efficient CM searches where the model can be fine tuned as needed to vary the search for specific objectives.

\section{Materials and Methods}

\subsection{Drosophila Sequence and Structure Prediction}

Figure 1 summarizes our data mining approach and illustrates the CM methodology utilized. We obtained eight roX1 RNA sequences experimentally (i.e., non-predicted) from Drosophila species (i.e., D. ananassae, D. erecta, D. melanogaster, D. mojavensis, D. pseudoobscura, D. simulans, D. virilis and D. yakuba) [7] (Table 1). In all cases, the sequences were obtained in raw format and were set up in standard FASTA format. Sequences were assigned arbitrary names as follows: in columns 1, 2 and 3, "yp1"; and in columns 4 and 5, a sequential 2-digit number. Starting and ending positions for all sequences were described in FASTA notation as "start:end" where "start" represents the first numeric position and "end" represents the last numeric position. For each of the original sequences, "start" had the value of 1 and "end" had the value of the length of the original sequence. The formatting of the starting and ending positions for each sequence was made compatible with RSmatch [16] so that when subsequences were extracted, the original FASTA annotation was preserved and additional subsequence position information was inserted for sequence tracking purposes.

As an illustration, the annotation for one FASTA sequence, named yp101, which was input to the RSmatch slide-and-fold process is shown here: ">yp101 (1:3493) droana rox1". Note that the length of this roX1 gene 
Table 1

Drosophila roX1 Sequences Used in This Study

\begin{tabular}{|c|c|c|c|c|c|}
\hline Species & Length & Secondary Structures* & Similarities $\diamond$ & FlyBase Region & Region Coordinates $^{\S}$ \\
\hline D. ananassae & 3,493 & 77 & 5,666 & scaffold_13117 & $\begin{array}{l}695557-693154 \\
693089-692300 \\
692143-692065 \\
692247-692215\end{array}$ \\
\hline D. erecta & 3,462 & 98 & 6,785 & scaffold_4690 & $\begin{array}{l}1139892-1137083 \\
1140318-1139928 \\
1137036-1136857\end{array}$ \\
\hline D. melanogaster & 3,468 & 106 & 7,249 & $\mathrm{X}$ & $\begin{array}{l}3755987-3754338 \\
3754043-3753143 \\
3756379-3756024 \\
3754304-3754082 \\
3753108-3752929\end{array}$ \\
\hline D. mojavensis & 3,768 & 99 & 6,864 & scaffold_6328 & $\begin{array}{c}3900419-3899115 \\
3901467-3900566 \\
3901937-3901499 \\
3902390-3902000 \\
3898736-3898624 \\
3898929-3898874 \\
3898845-3898810 \\
3947396-3947375 \\
246881-246900 \\
700541-700522\end{array}$ \\
\hline D. pseudoobscura & 3,469 & 92 & 6,385 & $\mathrm{XL}$ _group $1 \mathrm{e}$ & $\begin{array}{c}6901185-6898994 \\
6898915-6897910 \\
6897801-6897717 \\
1352025-1352045 \\
10880750-10880730 \\
476212-476239 \\
2910133-2910114\end{array}$ \\
\hline D. simulans & 3,439 & 101 & 7,049 & $\mathrm{X}$ & $\begin{array}{l}2761962-2759151 \\
2762379-2761996 \\
2759122-2758943 \\
9903425-9903446\end{array}$ \\
\hline D. virilis & 3,623 & 97 & 6,854 & scaffold_13042 & $\begin{array}{l}4639617-4638455 \\
4637622-4636608 \\
4638333-4637894 \\
4636532-4636064 \\
4637736-4637672 \\
4637835-4637787 \\
4636035-4635995 \\
4638396-4638367\end{array}$ \\
\hline D. yakuba & 3,433 & 103 & 7,425 & $\mathrm{X}$ & $\begin{array}{l}4658396-4661828 \\
3710814-3710795 \\
\end{array}$ \\
\hline Total & & 773 & 54,277 & & \\
\hline
\end{tabular}

* Secondary structures were predicted by the Vienna RNA package.

$\diamond$ Similarities with other Drosophila species were computed by RSmatch.

$\S$ Region and region coordinate information were obtained from FlyBase (http://www.flybase.org). 
Table 2

Illustration of Drosophila Sequences Used in the Creation of a CM

\begin{tabular}{|c|c|}
\hline \# STOCKHOLM 1.0 & \\
\hline dme_rox1:3102-3165 & GGUUCGUGUUUCGGAAAACGCAUUAAAAGGCGUAAUUUUAAAUCGUUUUCCGAAAUGGGA \\
\hline dsi_rox1:3079-3142 & GGUUCGUGUUUCGGAAAACGCUCUAAAAGGCGCAAUUUUAAAUCGUUUUCCGAAAUGGGA \\
\hline dya_rox1:3080-3143 & GGUUCGUGUUUCGGAAAACGCACUAAAAGGCGUAGUUUUGAAUCGUUUUCCGAAAUGGGA \\
\hline$\#=G C$ SS_cons & 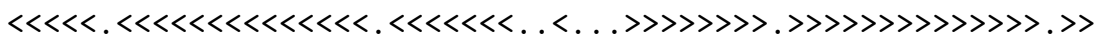 \\
\hline dme_rox1:3102-3165 & AUCA \\
\hline dsi_rox1:3079-3142 & AUCA \\
\hline dya_rox1:3080-3143 & AUCA \\
\hline$\#=$ GC SS_cons & $\gg>$ \\
\hline // & \\
\hline
\end{tabular}

The alignment is shown in Stockholm format. The numeric range following the species code represents the portion of the roX1 gene from which the sequence was extracted. The CM subsequently created was used in this study to search for other novel roX1 conserved domains.

sequence is 3,493 nucleotides (nt). RSmatch extracted subsequences from the original yp101 sequence and produced properly annotated FASTA format sequences such as this: ">yp101:51-150 (1:3493) droana rox1". Note that this annotation clearly represents a 100 nt sequence which was extracted from positions 51 through 150 of the original yp101 sequence. All of the original FASTA annotation information was retained. Providing position information in the annotation of the extracted subsequence is a critical function performed by RSmatch.

In a similar manner, all eight Drosophila roX 1 sequences evaluated for this work were annotated for compatibility with RSmatch, thus preserving subsequence positions. RSmatch slide-and-fold process was run with the following parameters: sequence size $=100$; overlap size $=50$; minimum free energy $=0$. We prepared RNA structures by the "slide and fold" method, as previously described [16]. Briefly, for each sequence, we took $100 \mathrm{nt}$ subsequences at every $50 \mathrm{nt}$ position from $5^{\prime}$ to $3^{\prime}$ resulting in consecutive subsequences overlapping with one another on a 50-nt segment. Subsequences shorter than $100 \mathrm{nt}$, e.g., at the $5^{\prime}$ or $3^{\prime}$ ends, were also kept. We then folded all of the subsequences using the RNAsubopt function in the Vienna RNA package [17] with the setting "-e 0". With this setting, multiple structures with the same minimum energy can be generated. Using this method, we obtained 773 structures from the eight Drosophila roX1 sequences.

\subsection{RNA Structure Comparison}

Pairwise comparisons of all RNA structures were carried out by RSmatch [16], with the "dsearch" function and default scoring matrices for single-stranded (ss) and doublestranded (ds) regions. Specifically, nucleotide match scores were 1 and 3 in ss and ds regions, respectively; and mismatch scores were -1 and 1 , in ss and ds regions, respectively. The gap penalty was -6 for both ss and ds regions. This scoring scheme in effect gave more weight on matches in ds regions than those in ss regions. We extracted three unduplicated FASTA sequences from high-scoring pairwise alignments.

\subsection{Creation}

The Mxscarna [18] package was used to align sequences for the CM used in the study. The resulting alignment was rendered in the Stockholm format with predicted structure annotation (Table 2). This alignment was input to the Infernal package utility cmbuild to create a CM. Figure 2 shows the consensus secondary structure of the sequence alignment used to create our CM [19].

\subsection{Drosophila Sequence Database}

The CM search utility "cmsearch" was run against a database of Drosophila FASTA sequences. The genomes from 12 Drosophila species (i.e., D. ananassae, D. erecta, D. grimshawi, D. melanogaster, D. mojavensis, D. persimilis, D. pseudoobscura, D. sechellia, D. simulans, D. virilis, D. willistoni and D. yakuba) were downloaded from Indiana University's FlyBase database (Table 3). Most Drosophila genomes have not been annotated into clearly defined chromosomes in FlyBase. As organismal research and sequencing efforts continue, we expect that the genomes of these 12 species of Drosophila will be fully annotated regarding specific chromosome identification.

\subsection{Search}

The Infernal package (version 1.0) utility "cmsearch" was used to locate structures in genomes with high degree of probability of matching the constructed CM [12]. To improve computational efficiency, large FASTA sequences were split into smaller, overlapping subsequences to facilitate independent parallel searching without negatively impacting results.

Figure 3 illustrates predicted secondary structures identified by the CM search as having high similarity relative to the profile that the CM represents. Out of the seven structures shown in Fig. 3, only the coordinates for $D$. sechellia's roX1 are not available for comparison. Given the structural similarity and high score result of the CM search, we propose that the D. sechellia sequence 


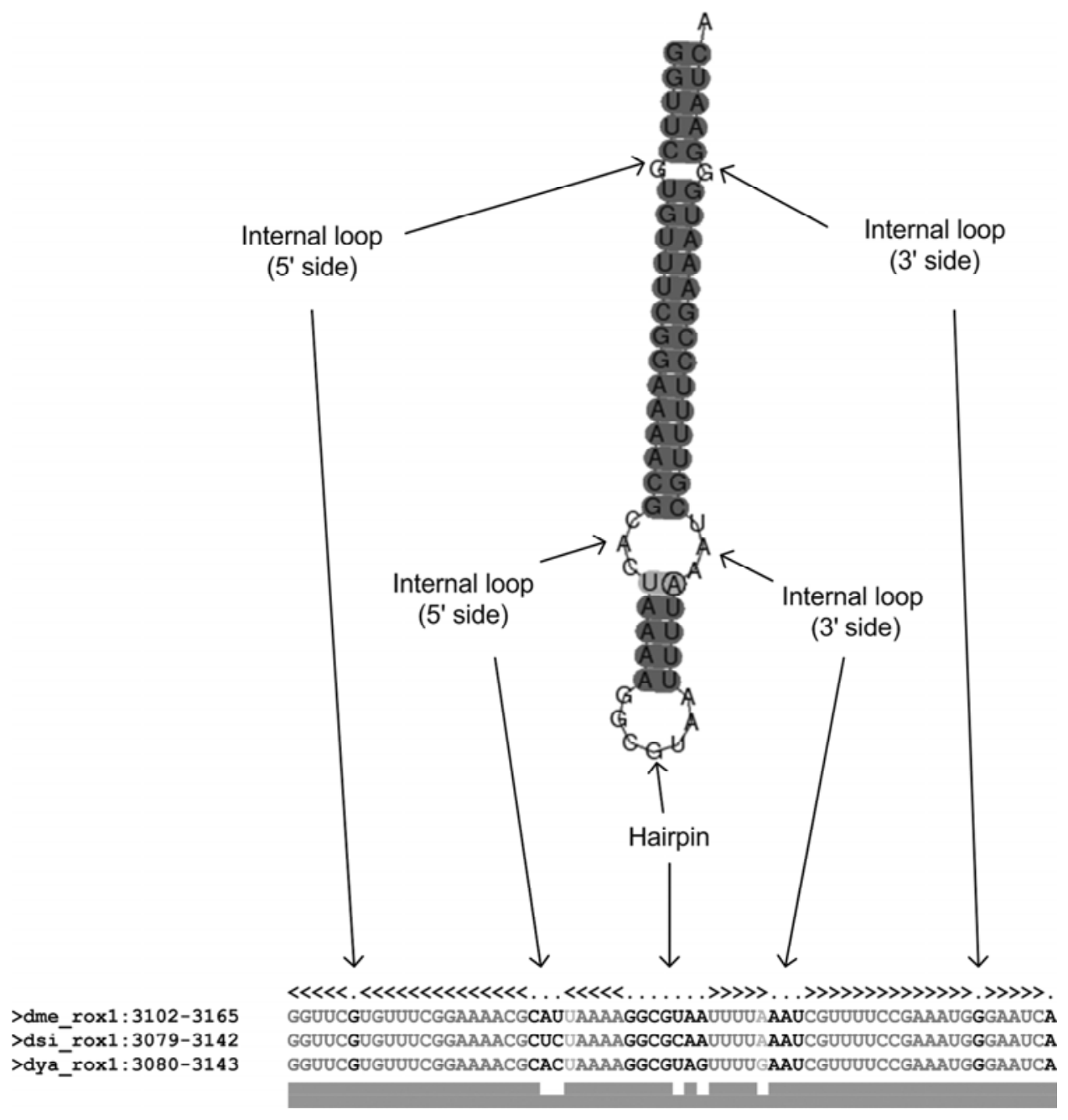

Figure 2. Illustration of the substructures of the RNA secondary structure representing the consensus structure of the alignment of three Drosophila roX1 sequences from species D. melanogaster, D. simulans and D. yakuba.

discovered is likely to represent roX1 functional domain characteristics.

\section{Results}

\subsection{Mining roX1 RNA Where the Presence of $r o X 1$ is Known}

Our purpose was to identify functional structure elements in genomes of Drosophila species in which the presence of roX1 has been experimentally demonstrated. To an extent, our strategy was similar to that recently proven successful by Khaladkar and others [16, 20-22]. First, we obtained eight sequences of roX1 RNA transcripts (Table 1). We then used a "slide and fold" method to construct RNA structures, as described in Methods. In this approach, subsequences 100-nt long or shorter were folded according to their thermodynamic properties using the Vienna RNA package [17]. Adjacent subsequences were overlapped by $50 \mathrm{nt}$. With this method, one can predict RNA structures accurately and efficiently for two reasons: (1) prediction of small ribonucleotide structures is more accurate and efficient than for large ones; and (2) structures with a size smaller than $50 \mathrm{nt}$ were folded twice as subsequences of two different larger structures, further increasing the probability of obtaining accurate RNA structure predictions. We also used a setting in the Vienna package that yielded multiple RNA structure predictions with the same minimum free energy for a given sequence to further improve folding accuracy. This step resulted in 773 predicted RNA structures.

We then carried out species versus species pairwise comparisons using all 773 predicted RNA structures. To make our approach computationally efficient, we ran each alignment as a process independent of all others on a high performance computing (HPC) cluster at the New Jersey Institute of Technology, leveraging emerging grid computing capabilities [23]. This HPC system, a Sun Microsystems Discovery cluster, has 112 AMD Opteron dual-core Linux nodes with $2 \mathrm{~GB}$ of RAM per node. The operating system used was Red Hat Enterprise Linux AS release 4 Update 8 . In this manner, approximately 520,000 pairwise alignments were completed in less than $5 \mathrm{~min}$, whereas we would have expected this process to take several 
Table 3

Description of 12 Drosophila Genomes Downloaded from FlyBase [29]

\begin{tabular}{|l|c|l|l|r|l|r|r|r|}
\hline Species & Release \# & $\begin{array}{l}\text { Release } \\
\text { date }\end{array}$ & Nucleotides & $\begin{array}{c}\text { Original } \\
\text { Sequences }\end{array}$ & $\begin{array}{l}\text { Region } \\
\text { Annotations }\end{array}$ & $\begin{array}{c}\text { Original } \\
\text { Files }\end{array}$ & $\begin{array}{c}\text { Modified } \\
\text { Sequences }\end{array}$ & $\begin{array}{c}\text { Modified } \\
\text { Files }\end{array}$ \\
\hline D.ananassae & 1.3 & 24Jul08 & $230,993,012$ & 13,749 & $\mathrm{Sc}$ & 1 & 13,809 & 128 \\
\hline D.erecta & 1.3 & 24Jul08 & $152,712,140$ & 5,124 & $\mathrm{Sc}$ & 1 & 5,183 & 81 \\
\hline D.grimshawi & 1.3 & 24Jul08 & $200,467,819$ & 17,440 & $\mathrm{Sc}$ & 1 & 17,502 & 121 \\
\hline D.melanogaster & 5.18 & 16May09 & $130,430,583$ & 7 & $\mathrm{Ch}$ & 7 & 69 & 70 \\
\hline D.mojavensis & 1.3 & 24Jul08 & $193,826,310$ & 6,841 & $\mathrm{Sc}$ & 1 & 6,916 & 106 \\
\hline D.persimilis & 1.3 & 24Jul08 & $188,374,079$ & 12,838 & $\mathrm{Sc}$ & 1 & 12,874 & 121 \\
\hline D.pseudoobscura & 2.4 & 19May09 & $152,738,921$ & 4,896 & $\mathrm{Ch}, \mathrm{Sc}$ & 1 & 4,952 & 87 \\
\hline D.sechellia & 1.3 & 24Jul08 & $166,577,145$ & 14,730 & $\mathrm{Sc}$ & 1 & 14,768 & 108 \\
\hline D.simulans & 1.3 & 24Jul08 & $137,828,247$ & 10,005 & $\mathrm{Ch}, \mathrm{Sc}$ & 1 & 10,057 & 73 \\
\hline D.virilis & 1.2 & 24Jul08 & $206,026,697$ & 13,530 & $\mathrm{Sc}$ & 1 & 13,600 & 121 \\
\hline D. willistoni & 1.3 & 24Jul08 & $235,516,348$ & 14,838 & $\mathrm{Sc}$ & 1 & 14,907 & 155 \\
\hline D.yakuba & 1.3 & 24Jul08 & $165,693,946$ & 8,122 & $\mathrm{Ch}, \mathrm{Sc}$ & 1 & 8,179 & 88 \\
\hline Total & & & $2,161,185,247$ & 122,120 & & 18 & 122,816 & 1,259 \\
\hline
\end{tabular}

To improve computational efficiency, files containing more than $2 \mathrm{Mbp}$ were separated into smaller files by distributing whole sequences. In a case where a sequence was greater than $2 \mathrm{Mbp}$, the sequence was split into multiple overlapping segments. Overlap size is $5 \mathrm{~K}$ bases. Annotation: $\mathrm{Ch}=$ chromosome; $\mathrm{Sc}=$ Scaffold
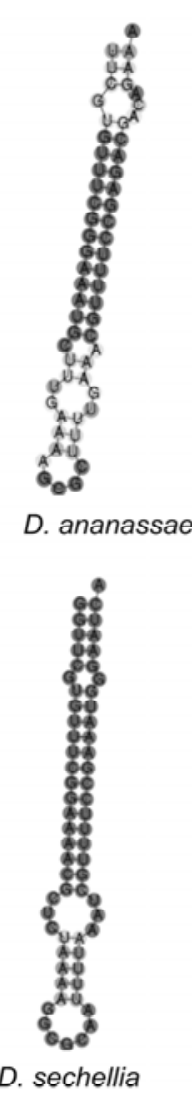

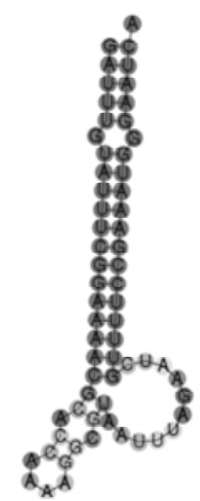

D. erecta

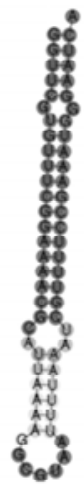

D. melanogaster

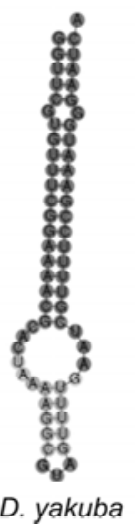

Figure 3. Samples of secondary structures of CM search results [12]. 
Table 4

Summary of Homologues Found in the Seven Drosophila Species

\begin{tabular}{|l|l|c|l|c|c|c|}
\hline ID & Genome Searched & CM Score & FlyBase Region & Region Coordinates & Strand & Within roX1? \\
\hline 1 & D. ananassae & 32.26 & scaffold_13117 & $692432-692373$ & - & $\mathrm{Y}$ \\
\hline 2 & D. erecta & 72.78 & scaffold_4690 & $1137235-1137172$ & - & $\mathrm{Y}$ \\
\hline 3 & D. melanogaster & 88.84 & chromosome X & $3753295-3753232$ & - & $\mathrm{Y}$ \\
\hline 4 & D. pseudoobscura & 29.4 & Unknown_group_410 & $14965-14898$ & - & $\mathrm{N}$ \\
\hline 5 & D. pseudoobscura & 29.11 & Unknown_group_260 & $63165-63089$ & - & $\mathrm{N}$ \\
\hline 6 & D. pseudoobscura & 28.28 & XL_group1e & $6898105-6898042$ & - & $\mathrm{Y}$ \\
\hline 7 & D. sechellia & 88.1 & scaffold_4 & $2954091-2954154$ & + & $\mathrm{N} / \mathrm{A}$ \\
\hline 8 & D. simulans & 88.1 & chromosome X & $2759303-2759240$ & - & $\mathrm{Y}$ \\
\hline 9 & D. yakuba & 88.69 & chromosome X & $4661475-4661538$ & + & $\mathrm{Y}$ \\
\hline
\end{tabular}

hours using a single-processor approach. Each comparison yielded an alignment score. We then selected a group of three structures that were scored similarly and whose sequence lengths were at least $40 \mathrm{nt}$. At this step, RNA structures were obtained from $D$. melanogaster, D. simulans and D. yakuba.

\subsection{Creation and Search}

We created a CM from this group of structures by first aligning the sequences into the Stockholm format (Table 2) and then executing the cmbuild utility. The complete genomes of eight Drosophila species for which the presence of roX1 ncRNA transcripts has been confirmed were used as targets in CM searches. All complete genomes used in this study were obtained from Indiana University's FlyBase database (http://www.flybase.org) [21]. These genomes were the most current releases at the time the study was conducted (Table 3). A CM search located the roX1 genes precisely where they were known to be present in six Drosophila species, i.e., D. ananassae, D. erecta, D. melanogaster, D. pseudoobscura, D. simulans and D. yakuba. However, the CM search failed to locate the known roX1 ncRNAs on the remaining two Drosophila species, i.e., D. mojavensis and D. virilis (Table 4). In five of the six successful searches, the highest scoring search result represented a sequence within the known range of the roX1 genomic coordinates for that species. The sixth successful search, on D. pseudoobscura, produced the third highest scoring search result that represented a sequence within the known range of the roX1 genomic coordinates for that species. We hypothesize that the two highest scores for D. pseudoobscura represent sequences with conserved roX1 functionality.

To make our searching approach computationally efficient, we separated the downloaded genome files into smaller files with approximately two megabase pairs (Mbp) per file maintaining small FASTA sequences intact. FASTA sequences larger than $2 \mathrm{Mbp}$ were split into smaller FASTA sequences which overlapped one another by 5 kilobase pairs (Kbp) to prevent loss of accuracy in the study. This approach is similar to the slide-and-fold approach described in the RSmatch discussion in this paper. We performed concurrent identical cmsearch runs on different genome segments using NJIT's HPC cluster. In this manner, a $\mathrm{CM}$ search of an entire genome required only about $10 \mathrm{~min}$, whereas we would have expected a genome search to take several hours using a single-processor approach.

\subsection{Mining roX1 RNA Where the Presence of $r o X 1$ is Unknown}

We wanted to identify functional structure elements in genomes of the four Drosophila species in which the presence of roX1 transcripts has not been confirmed, namely, D. grimshawi, D. persimilis, D. sechellia and D. willistoni. We downloaded the most current release of these complete genomes from the FlyBase database. We used the same CM to search for presence of roX1 functional domains. While scoring results were not significant for three of the four species, we received a strong score result from the CM search on the D. sechellia genome (Tables 4 and 5). We propose that this high score indicates strong evidence of a roX1 functional domain in a specific area of the $D$. sechellia genome, namely scaffold_4. Furthermore, in spite of the D. sechellia's incomplete annotation, this result might indicate that this region of the genome may be located in the $\mathrm{X}$ chromosome of $D$. sechellia. These findings need to be experimentally confirmed.

To investigate possible roX1 homology between species, we obtained roX1 gene sequences FBgn0019661 (for D. melanogaster) and FBgn0255860 (for D. sechellia) from the FlyBase database and performed a pairwise alignment on the two sequences. We used the program DiAlign [24] with the "-n" option for nucleic acid sequence comparison. The result indicated a $94 \%$ similarity between the two gene sequences indicating high probability of conserved roX1 functionality between the two species.

We have designed a systematic and computationally efficient approach to mine roX1 RNA structure elements conserved in Drosophila species. This approach consists of three major steps: (1) comparison of RNA structures among all roX1 RNAs; (2) selection of RNA structure 
Table 5

Homologous RNA Sequences Found in Drosophila Species

\begin{tabular}{|c|c|c|}
\hline ID & RNA Sequence & Length \\
\hline 1 & UUC - GUGUUUCGGGAAAUGCUUUGAAAAGCG - CUUUUGAAACGUUUUCCGAGACGACAGAAA & 60 \\
\hline 2 & GAUUUGUAUUUCGGAAAACGCACCAAAAGGCGUAAUUUAGAAUCGUUUUCCGAAAUGGGAAUCA & 64 \\
\hline 3 & GGUUCGUGUUUCGGAAAACGCAUUAAAAGGCGUAAUUUUAAAUCGUUUUCCGAAAUGGGAAUCA & 64 \\
\hline 4 & GACCACUCCUUCGGGUACCUCAAAAAAAaagGGCAUAGgUAUUUGGGAGGUACCCGAAGGAGUGGUCU & 68 \\
\hline 5 & UCCACACGUUUCCAACUUCGUUUCCACACGC $* * * * * * * * * * * * * *$ GUGUGGAAACGAAGUUGGAAACGCguGUGGAA & 77 \\
\hline 6 & CGUUCGGGUUUCGGAAAACGCGUCGA $* * * * * * * * * * * * * *$ UUGAAACGUUUUCCGAAAC-AGAA —A & 64 \\
\hline 7 & GGUUCGUGUUUCGGAAAACGCUCUAAAAGGCGCAAUUUUAAAUCGUUUUCCGAAAUGGGAAUCA & 64 \\
\hline 8 & GGUUCGUGUUUCGGAAAACGCUCUAAAAGGCGCAAUUUUAAAUCGUUUUCCGAAAUGGGAAUCA & 64 \\
\hline 9 & GGUUCGUGUUUCGGAAAACGCACUAAAAGGCGUAGUUUUGAAUCGUUUUCCGAAAUGGGAAUCA & 64 \\
\hline
\end{tabular}

An asterisk $\left(^{*}\right)$ indicates a base that is left unaligned with a CM counterpart; a minus sign (-) indicates that no base is present to align with a CM counterpart (not included in the sequence length) and a lowercase letter represents a base on the genome that is added with respect to the CM.

groups significantly associated with those in other species and (3) utilization of a highly regarded structure-searching methodology (i.e., CMs) which, in addition to being highly sensitive and specific, is also very flexible. The statistical representation of a cluster of RNA structures can be fine tuned as needed by adding or removing structures from the cluster. Using parallel processing contributes to overcoming the burden of lengthy processing times. We applied this method to mining small RNA structures chiefly because they can be more accurately forecast by those RNA prediction programs that only use thermodynamic parameters. As more powerful RNA structure prediction programs become available, particularly those reliant on phenetics for structure prediction, this approach can be extended to larger RNA structures.

\subsection{Tool Comparison: Infernal versus BLAST}

For the purpose of tool-effectiveness analysis, we wanted to see how a simple BLAST search might perform compared with Infernal when searching for conserved structural motifs. As BLAST is not designed to detect base pairing so critical to form an RNA secondary structure, we expected Infernal to perform better than BLAST. Table 6 presents the results of a simple test that confirmed our expectations. We used each of the three sequences from our CM and used FlyBase BLAST to search for homologues in the complete genomes of all 12 Drosophila species downloaded from FlyBase. Every homologue detected by BLAST was also detected by Infernal. However, BLAST failed to detect roX1 evidence in D. ananassae and D. pseudoobscura, while such evidence was detected by Infernal. This simple experiment provides an insight into the complexity involved in the mining of ncRNA motifs.

\subsection{Evaluation of the Drosophila Genus Complex}

By conducting homology search on a complete genome, one can confirm whether a functional domain is present throughout the genome of a species or rather at specific sequence locations (i.e., genomic coordinate ranges) within a specific genomic region such as a chromosome, a scaffold, etc. A stem-loop structure was previously predicted in roX1 RNA on the X chromosome of D. melanogaster [25], and we subsequently determined that this structure is conserved in several species of Drosophila [7]. Our study confirms that among seven different Drosophila species, the roX1 functional domain is only present on the $\mathrm{X}$ chromosome but also absent in any chromosome other than X. As genome annotation matures and "scaffold" regions are translated into "chromosome" regions, we will see whether this observation continues to hold.

\section{Conclusion}

In this study, we demonstrated that the tools RSmatch and Infernal are effective in identifying novel ncRNAs. Homology searching is a most ubiquitous undertaking in bioinformatics, yet some of the most popular homology search methods such as BLAST and FASTA, are often the least accurate [26]. Homology search tasks are more challenging for ncRNAs than a regular sequence homology search. This stems from an evolutionary perspective: ncRNA secondary structures due to intramolecular base pairs are conserved to a higher degree with respect to their primary structure, i.e., their nucleotide sequence.

An Infernal search requires a large amount of computer time [12]. For instance, Freyhult et al. [26] estimated that with a search query for a tRNA (a type of ncRNA), Infernal would take about 96 days to search the entire human genome on a single processor. Innovative methodologies including HMM filtering and sequence-based heuristics [27, 28] have been employed as appropriate to improve computational efficiency. In this study, as described, parallel processing with an HPC cluster was utilized for improved throughput. 
Table 6

FlyBase BLAST Results Using twelve Drosophila Genomes As the Targets With Each of Three CM Sequences Used As a Search Query

\begin{tabular}{|c|c|c|c|c|}
\hline $\begin{array}{l}\text { Species Genome } \\
\text { Searched }\end{array}$ & $\begin{array}{l}\text { D. melanogaster } \\
\text { Sequence Query }\end{array}$ & $\begin{array}{c}\text { D. simulans } \\
\text { Sequence Query }\end{array}$ & $\begin{array}{c}D . y a k u b a \\
\text { Sequence Query }\end{array}$ & $\begin{array}{c}\text { Infernal ro } X 1 \\
\text { Hit Score }\end{array}$ \\
\hline D. ananassae & no hit & no hit & no hit & 32.26 \\
\hline D. erecta & $\begin{array}{c}1137227(-) \\
1137172 \\
52 / 56(93 \%)\end{array}$ & $\begin{array}{c}1137227(-) \\
1137172 \\
51 / 56(91 \%)\end{array}$ & $\begin{array}{c}1137227(-) \\
1137172 \\
53 / 56(95 \%)\end{array}$ & 72.78 \\
\hline D. melanogaster & $\begin{array}{c}3753295(-) \\
3753232 \\
64 / 64(100 \%)\end{array}$ & $\begin{array}{c}3753295(-) \\
3753232 \\
61 / 64(95 \%)\end{array}$ & $\begin{array}{c}3753295(-) \\
3753232 \\
61 / 64(95 \%)\end{array}$ & 88.84 \\
\hline D. mojavensis & no hit & no hit & no hit & no hit \\
\hline D. pseudoobscura & no hit & no hit & no hit & $28.28(3 \mathrm{rd})$ \\
\hline D. simulans & $\begin{array}{c}2759303(-) \\
2759240 \\
61 / 64(95 \%)\end{array}$ & $\begin{array}{c}2759303(-) \\
2759240 \\
64 / 64(100 \%)\end{array}$ & $\begin{array}{c}2759303(-) \\
2759240 \\
60 / 64(94 \%)\end{array}$ & 88.1 \\
\hline D. virilis & no hit & no hit & no hit & no hit \\
\hline D. yakuba & $\begin{array}{c}4661475(-) \\
4661538 \\
61 / 64(95 \%)\end{array}$ & $\begin{array}{c}4661475(-) \\
4661538 \\
60 / 64(94 \%)\end{array}$ & $\begin{array}{c}4661475(-) \\
4661538 \\
64 / 64(100 \%)\end{array}$ & 88.69 \\
\hline D. grimshawi & $2 @ 18 n t$ & $1 @ 17 n t$ & $2 @ 17 n t$ & $26.13(?)$ \\
\hline D. persimilis & $1 @ 21 n t$ & $1 @ 17 n t$ & $1 @ 19 n t$ & $39.51(?)$ \\
\hline D. sechellia & $1 @ 61 n t$ & $1 @ 64 n t$ & $1 @ 64 n t$ & $88.1(?)$ \\
\hline D. willistoni & $2 @ 19 n t$ & $2 @ 18 n t$ & $1 @ 18 n t$ & $35.19(?)$ \\
\hline
\end{tabular}

Notes: Infernal search results shown for comparison. (?)= roX1 hit unknown; "no hit" = not found within roX1 coordinates.

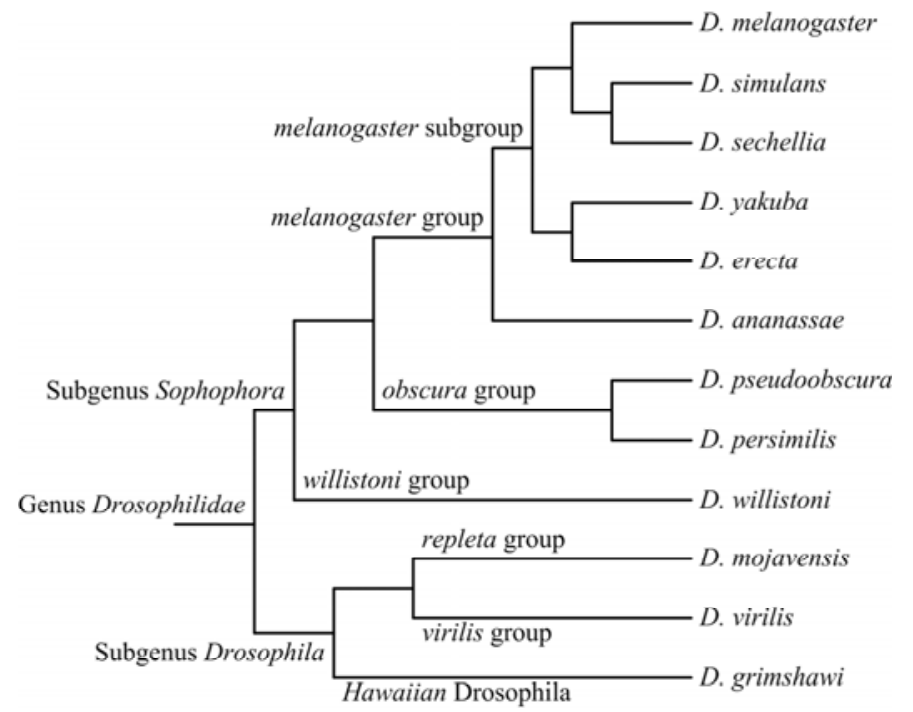

Figure 4. Phylogram of the genus Drosophila showing the evolutionary relationships among the 12 sequenced Drosophila species. Distances between nodes do not reflect base substitution span. Adapted from Stark et al. [9], and FlyBase [29].
We evaluated the whole genomes of all 12 species of Drosophila that have been completely sequenced to date. Figure 4 displays a phylogram of the genus Drosophila complex, which will contribute to understanding the phylogenetic relationships among the 12 Drosophila species evaluated in this study. All 12 species are believed to have a common ancestor that existed about 40 million years ago [7]. Phylogenetic relationships are based on the premise that species which evolved most recently will have more genetic similarities among them than those species that evolved much earlier. As a result of our study, the presence of the roX 1 ncRNA is confirmed as previously reported by other authors in six Drosophila species [7]. In addition, we found strong evidence of the presence of $\operatorname{roX} 1$ in $D$. sechellia which, to the best of our knowledge, has not been previously reported. Through such comparative genomics, each discovery of similarities or differences among closely related species within a genus contributes to unravelling the mysteries of evolution and inborn errors of metabolism leading to human disease.

In summary, we expect that further studies will verify that ro $X 1$ ncRNA structures can be predicted where there is evidence of roX1 functionality. We also expect that 
this bioinformatics study will lay ground work for similar accurate and efficient ncRNA mining in other organisms.

\section{References}

[1] S.R. Eddy, Non-coding RNA genes and the modern RNA world, Nature Reviews Genetics, 2, 2001, 919-929.

[2] G. Storz, An expanding universe of noncoding RNAs, Science, 296, 2002, 1260-1263

[3] J.S. Mattick \& I. V. Makunin, Non-coding RNA, Human Molecular Genetics, 15 Spec No 1, 2006, R17-R29.

[4] F.F. Costa, Non-coding RNAs: Lost in translation? Gene, 386, 2007, 1-10.

[5] Y. Park \& M.I. Kuroda, Epigenetic aspects of X-chromosome dosage compensation, Science, 293(5532), 2001, 1083-1085.

[6] Y. Park, R.L. Kelley, H. Oh, M.I. Kuroda, \& V.H. Meller, Extent of chromatin spreading determined by roX RNA recruitment of MSL proteins, Science, 298(5598), 2002, 1620-1623.

[7] S. Park, Y.I. Kang, J.G. Sypula, J. Choi, H. Oh, \& Y. Park, An evolutionarily conserved domain of roX2 RNA is sufficient for induction of H4-Lys16 acetylation on the Drosophila X chromosome, Genetics, 177(3), 2007, 1429-1437.

[8] S. Park, M.I. Kuroda, \& Y. Park, Regulation of histone H4 Lys16 acetylation by predicted alternative secondary structures in roX noncoding RNAs. Molecular and Cellular Biology, 28(16), 2008, 4952-4962.

[9] A. Stark, et al., Discovery of functional elements in 12 Drosophila genomes using evolutionary signatures. Nature, 450, 2007, 219-232.

[10] A. Wang, W. Ruzzo, \& M. Tompa, How accurately is ncRNA aligned within whole-genome multiple alignments? BMC Bioinformatics, 8, 2007, 417.

[11] R. Durbin, S. Eddy, A. Krogh, \& G. Mitchison, Biological sequence analysis: probabilistic models of proteins and Nucleic acids (Cambridge UK: Cambridge University Press 1998), 277-297.

[12] E.P. Nawrocki, D.L. Kolbe, \& S.R. Eddy, Infernal 1.0: Inference of RNA alignments, Bioinformatics, 25(10), 2009, 1335-1337.

[13] S. Griffiths-Jones, A. Bateman, M. Marshall, A. Khanna, \& S.R. Eddy, Rfam: An RNA family database, Nucleic Acids Research, 31, 2003, 439-441.

[14] S. Griffiths-Jones, S. Moxon, M. Marshall, A. Khanna, S. R. Eddy, \& A. Bateman, Rfam: Annotating non-coding RNAs in complete genomes, Nucleic Acids Research, 33, 2005, D121D124.

[15] L.A. Kavanaugh \& F.S. Dietrich, Non-coding RNA prediction and verification in Saccharomyces cerevisiae, PLoS Genetcis 5(1), 2009, e1000321.

[16] J. Liu, J.T.L. Wang, J. Hu, \& B. Tian, A method for aligning RNA secondary structures and its application to RNA motif detection, BMC Bioinformatics 6, 2005, 89.

[17] I.L. Hofacker, Vienna RNA secondary structure server, Nucleic Acids Research 31, 2003, 3429-3431.

[18] Y. Tabei, H. Kiryu, T. Kin, \& K. Asai, A fast structural multiple alignment method for long RNA sequences, $B M C$ Bioinformatics, 9, 2008, 33.

[19] A.R. Gruber, R. Neubock, I.L. Hofacker, \& S. Washietl, The RNAz web server: Prediction of thermodynamically stable and evolutionarily conserved RNA structures, Nucleic Acids Research, 35, 2007, W335-W338.

[20] M. Khaladkar, J. Liu, D. Wen, J.T.L. Wang, \& B. Tian, Mining small RNA structure elements in untranslated regions of human and mouse mRNAs using structure-based alignment, BMC Genomics, 9, 2008, 189.

[21] M. Khaladkar, V. Patel, V. Bellofatto, J. Wilusz, \& J.T.L. Wang, Detecting conserved secondary structures in RNA molecules using constrained structural alignment, Computational Biology and Chemistry, 32, 2008, 264-272.

[22] M. Khaladkar, V. Bellofatto, J.T.L. Wang, B. Tian, \& B.A. Shapiro, RADAR: A web server for RNA data analysis and research, Nucleic Acids Research, 35, 2007, W300-W304.

[23] L. Peng, L.K. Ng, \& S. See, YellowRiver: A flexible high performance cluster computing service for grid, Proceedings Eighth IEEE International Conference on High-Performance Computing in Asia-Pacific Region, 2005, 553-558.
[24] A. Subramanian, M. Kaufmann, \& B. Morgenstern, DIALIGNTX: Greedy and progressive approaches for segment-based multiple sequence alignment, Algorithms for Molecular Biology, 3, 2008, 6 .

[25] C. Stuckenholz, V.H. Meller, \& M.I. Kuroda, Functional redundancy within roX1, a noncoding RNA involved in dosage compensation in Drosophila melanogaster, Genetics, 164, 2003, 1003-1014.

[26] E.K. Freyhult, J.P. Bollback, \& P.P. Gardner, Exploring genomic dark matter: A critical assessment of the performance of homology search methods on noncoding RNA, Genome Research, 17(1), 2007, 117-125.

[27] Z. Yao, Z. Weinberg, \& W.L. Ruzzo, CMfinder - A covariance model based RNA motif finding algorithm, Bioinformatics, 22(4), 2006, 445-452.

[28] Z. Weinberg \& W.L. Ruzzo, Sequence-based heuristics for faster annotation of non-coding RNA families, Bioinformatics, 22, 2006, 35-39.

[29] The FlyBase Consortium, FlyBase - The Drosophila database, Nucleic Acids Research, 22, 1994, 3456-3458.

\section{Biographies}

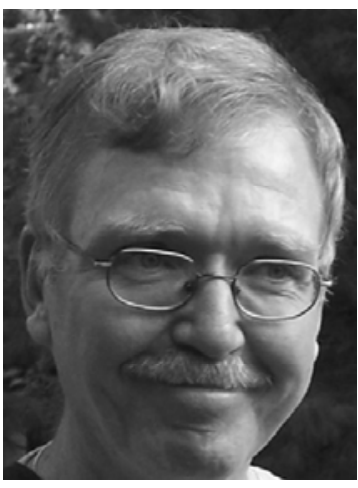

Kevin Byron received his B.S. degree in Computer Science from New Jersey Institute of Technology, USA in 1983 and his M.S. degree in computer science from Stevens Institute of Technology, USA in 1987. He is Director of Core Systems and Operations at New Jersey Institute of Technology, USA. He is currently a Ph.D. candidate in Computer Science at New Jersey Institute of Technology, USA. His research interests are bioinformatics and Drosophila genomic data mining.

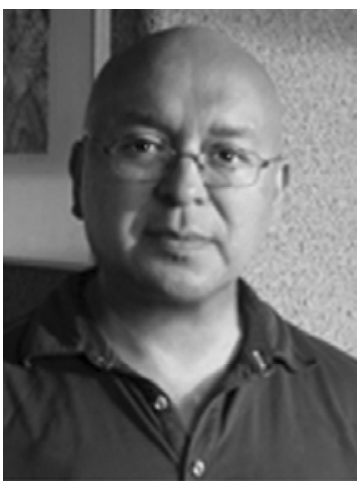

Miguel Cervantes-Cervantes studied Biology as an undergraduate at the Instituto Politécnico Nacional in Mexico City, where he also received a Master of Science degree in Biochemistry. His interest in the inner workings of the plant cell took him to the Waksman Institute of Rutgers, The State University of New Jersey, where he obtained a Ph.D. in biochemistry in 1991. After training as a post-doctoral associate in plant cell biology at Rutgers-Newark, he worked at the City University of New York from 1996 to 2007. Currently, Miguel is the coordinator of undergraduate studies at the Federated Department of Biological Sciences of Rutgers-Newark and the New Jersey Institute of Technology. He continues doing research on cereal seed germination and metabolic engineering, focusing on the isoprenoid biosynthetic genes and enzymes, and has started utilizing bioinformatics tools in his research on metabolic networks as well as in graduate courses in plant science. 


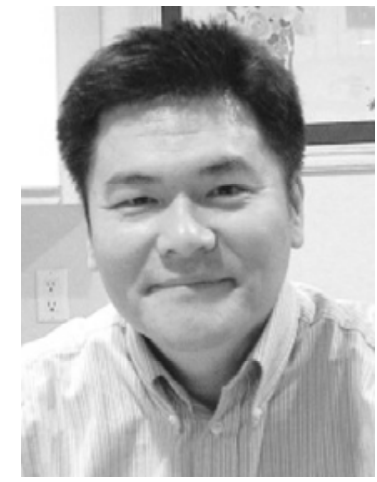

Jason T.L. Wang received a B.S. in Mathematics from National Taiwan University, Taipei, Taiwan, and a Ph.D. in Computer Science from the Courant Institute of Mathematical Sciences at New York University. $\mathrm{He}$ is a Professor of computer science and bioinformatics at the New Jersey Institute of Technology. His research interests include data mining, computational biology and bioinformatics. He has published over 120 peer-reviewed papers, 5 books, has been a program committee member of over 100 conferences, and is on the editorial board of 15 journals as well as the Executive Editor of the World Scientific book series on science, engineering and biology informatics. He is currently the principal investigator of an NSF-funded project on RNA genomics.

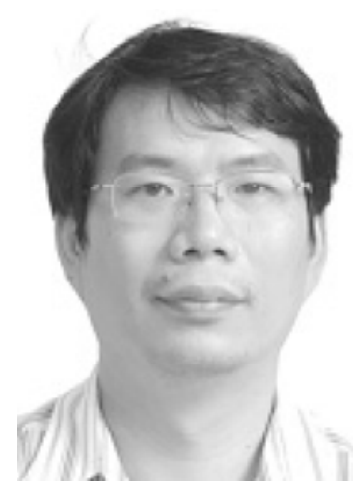

Wen-chang Lin received his B.S. degree in Agricultural Chemistry from National Taiwan University, Taiwan in 1986 and his Ph.D. degree in Molecular Biology and Microbiology from Case Western Reserve University, USA in 1991. After training as a Post-Doctoral Associate in tumor immunology and gene therapy at Pittsburgh Cancer Institute from 1991 to 1993, he returned to Taiwan in 1993 and joined Institute of Biomedical Sciences, Academia Sinica, Taiwan. He is currently an Associate Research Fellow at
Academia Sinica. Wen-chang's research interests include tumor progression and biomarker discovery; bioinformatic studies on human genome annotation and transcriptome data-mining (subtle wobble splicing); microRNA discovery and functional characterization.

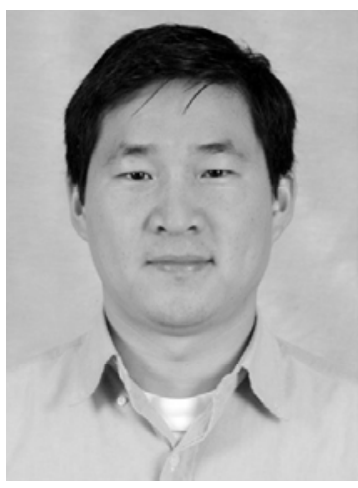

Yongkyu Park received a B.S. in Biology from Korea University, Seoul, Korea, and a Ph.D. in microbiology from Korea Advanced Institute of Science and Technology (KAIST) in 1999. From 1999 to 2004, he was a Postdoctoral Researcher in molecular genetics at Baylor College of Medicine, Houston, Texas, and then a Postdoctoral Researcher at Harvard Medical School, Boston, Massachusetts. He is currently an Assistant Professor in the Department of Cell Biology and Molecular Medicine of the New Jersey Medical School in the University of Medicine and Dentistry of New Jersey. His researcher interest is focused on noncoding RNA (ncRNA) in Drosophila. He is the principal investigator of an NSF-funded project on roles of ncRNA in global chromatin organization. 